\title{
Analysis of the magnetic coupling in binuclear systems. III. The role of the ligand to metal charge transfer excitations revisited
}

\author{
Carmen J. Calzado, ${ }^{1, a)}$ Celestino Angeli, ${ }^{2}$ David Taratiel, ${ }^{3}$ Rosa Caballol, ${ }^{3}$ and \\ Jean-Paul Malrieu ${ }^{4}$ \\ ${ }^{1}$ Departamento de Química Física, Universidad de Sevilla, \\ Profesor García González s/n, E-41012 Sevilla, Spain \\ ${ }^{2}$ Dipartimento di Chimica, Università di Ferrara, via Borsari 46, I-44100 Ferrara, Italy \\ ${ }^{3}$ Departament de Química Física i Inorgànica, Universitat Rovira i Virgili, \\ Marcel lí Domingo s/n, E-43007 Tarragona, Spain \\ ${ }^{4}$ Laboratoire de Physique et Chimie Quantiques, Université Paul Sabatier, \\ 118 Rte de Narbonne, 31062 Toulouse Cedex, France
}

(Received 26 March 2009; accepted 4 July 2009; published online 31 July 2009)

In magnetic coordination compounds and solids the magnetic orbitals are essentially located on metallic centers but present some delocalization tails on adjacent ligands. Mean field variational calculations optimize this mixing and validate a single band modelization of the intersite magnetic exchange. In this approach, due to the Brillouin's theorem, the ligand to metal charge transfer (LMCT) excitations play a minor role. On the other hand the extensive configuration interaction calculations show that the determinants obtained by a single excitation on the top of the LMCT configurations bring an important antiferromagnetic contribution to the magnetic coupling. Perturbative and truncated variational calculations show that contrary to the interpretation given in a previous article [C. J. Calzado et al., J. Chem. Phys. 116, 2728 (2002)] the contribution of these determinants to the magnetic coupling constant is not a second-order one. An analytic development enables one to establish that they contribute at higher order as a correlation induced increase in the LMCT components of the wave function, i.e., of the mixing between the ligand and the magnetic orbitals. This larger delocalization of the magnetic orbitals results in an increase in both the ferroand antiferromagnetic contributions to the coupling constant. (c) 2009 American Institute of Physics. [DOI: $10.1063 / 1.3185506]$

\section{INTRODUCTION}

An enormous effort has been devoted in the past to the understanding of the factors controlling the interactions between the spin moments on magnetic systems. This effort has been driven by the aim to rationalize the macroscopic magnetic behavior and to help in the design of new materials with particular properties. Since the seminal work of Anderson, ${ }^{1,2}$ many models have been proposed to explain magnetic exchange interactions. These models intend to express the magnetic coupling constant $J$ on the basis of a reduced number of parameters, which take into account the main physical effects of the coupling. Most of them only deal with the magnetic orbitals and the unpaired electrons in their simplest version with only one electron per metallic center. For $S=\frac{1}{2}$ binuclear systems, these one-band models reduce to two electrons on two magnetic orbitals $a$ and $b$, and $J$ can be expressed in the Anderson mechanism as a function of only three parameters: the direct exchange between the active orbitals $K_{a b}$, the one-electron transfer between metal centers $t_{a b}$, and the on-site Coulomb repulsion $U$,

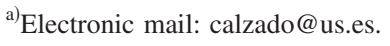

$$
J=2 K_{a b}-4 t_{a b}^{2} / U=J_{F}+J_{\mathrm{AF}},
$$

where the former term represents the ferromagnetic $(F)$ direct exchange contribution to the coupling and the latter takes into account the delocalization effects favoring the antiferromagnetic (AF) kinetic exchange contribution. This AF component is driven by the coupling between the valence bond (VB) neutral $N=\{|a \bar{b}|,|b \bar{a}|\}$ determinants and the VB ionic $I=\{|a \bar{a}|,|b \bar{b}|\}$ determinants. These configurations define a minimal valence complete active space (CAS).

The other models introduce also orbitals located on the ligands bridging the metallic centers, that is, a two-band model, recently revisited by Van den Heuvel and Chibotaru. ${ }^{3}$ The simplest version adds only one ligand orbital, resulting in a model with four electrons in three orbitals. In this case, the ligand to metal charge transfer (LMCT) states play a crucial role, mediating the coupling between the neutral and ionic VB determinants. Therefore, additional parameters need to be included, namely, the one-electron hopping integral between metal and ligand orbitals $t_{a l}$ or $t_{b l}$, the on-site ligand Coulomb repulsion $U_{l}$, and the ligand-to-metal charge transfer excitation energy $\Delta E_{\mathrm{CT}}$.

Simultaneous to the development of these models, $a b$ initio calculations have provided more and more accurate values of $J$ on systems of increasing complexity and size. Both density functional theory based approaches (DFT) and 
wave function-based methods have extensively been used and their performances and limitations discussed by several authors. ${ }^{4-6}$ Bencini ${ }^{7}$ recently gave an excellent review on the use of these computational tools. Together with numerical accuracy, the importance of these calculations lies in the fact that they have clarified some aspects of the factors governing the coupling. Wave function-based methods are especially useful for these interpretation purposes. In particular they have put in evidence that the $J$ values resulting from the action of the exact Hamiltonian on the model space are at least one order of magnitude smaller than the experiment or even of incorrect sign. Consequently, the physics of the coupling can neither be restricted to the interaction of the neutral and ionic VB determinants, as in one-band models, nor to their coupling with the LMCT states, as in two-band ones.

Different approaches have been used to analyze the physical contributions to the magnetic coupling beyond the active-electron only approximation. Particularly remarkable is the pioneering work carried out by de Loth et $a l^{8}{ }^{8}$ in the early 1980s and all the applications reported during the following decade. ${ }^{9-12}$ They have provided expressions for the perturbative evaluation of $J$ from the energy gap between the singlet and triplet states of binuclear $\mathrm{Cu}$ (II) complexes. They followed an idea developed by Malrieu ${ }^{13}$ in the 1960 s who showed that it was not necessary to calculate the total correlation energy for both singlet and triplet states when using perturbation theory. Inspired by this work, Broer and Maaskant ${ }^{14}$ performed early variational calculations including the same configurations on copper dinuclear complexes. Following the same ideas, Malrieu and co-workers ${ }^{15,16}$ developed the difference dedicated configuration interaction (DDCI) approach where the CI matrix only contains those configurations playing a role on the energy difference of the states involved in the coupling. Based on the same idea, Neese and co-workers ${ }^{17,18}$ recently developed and coded the spectroscopy oriented CI method focused to the study of transition metal complexes. Recently, Barone et al. ${ }^{19}$ also coded a modified DDCI2 version of the method and applied to organic biradicals. From a computational point of view the DDCI procedure is much more expensive than the perturbative evaluations of de Loth et al., ${ }^{8}$ but, in contrast, it is free of convergence problems and of the implicit arbitrariness related to the definition of the zeroth-order Hamiltonian. So far, the DDCI approach has been extensively employed in the evaluation of $J$ in molecular and solid state magnetic materials with a remarkable good agreement with experiment. ${ }^{20-32}$

Some years ago we took benefit of this methodology to analyze in depth the physical contributions to the magnetic coupling on a series of binuclear $\mathrm{Cu}$ (II) complexes. ${ }^{33}$ The use of the DDCI strategy allows not only to obtain quite accurate $J$ values but also to analyze the various physical effects by generating CI spaces of increasing lengths that include different types of determinants. We also reported a procedure to define valence effective Hamiltonians starting from the eigenenergies and eigenvectors of these CI calculations. ${ }^{34}$ Indeed, the procedure provides a scheme to return to the qualitative models with effective parameters, which incorporate not only the effects brought by the model space but also those beyond the active-electron approximation, which in most of the cases have their origin in the electronic correlation. After classifying the excited determinants according to the number of doubly occupied orbitals $(h)$ and virtual orbitals $(p)$ implied in the excitation processes on the CAS determinants, our results indicate that the main contributions to the coupling come from the following:

(i) The excitations involving one inactive occupied orbital $h$ and one inactive virtual orbital $p$. This $1 h-1 p$ class of excitations introduces spin polarization effects as well as the polarization of the ionic VB forms, whose main effect consists of lowering the effective energy of ionic configurations. Both effects are particularly important when they involve orbitals centered in the bridging ligands.

(ii) The $2 h-1 p$ and $1 h-2 p$ classes of excitations. Some of them can be considered as single excitations on the LMCT and metal to ligand charge transfer (MLCT) configurations, respectively. Their impact on the $J$ value is at least $20 \%-30 \%$ of the global value, even more than $50 \%$ in some cases. When this type of excitations is included in the CI expansions computed $J$ values are closer to the experiment.

Previous interpretation of the mechanism governing the effect of these later excitations on the magnetic coupling was essentially formulated in terms of an enhancement of the effective hopping integral $t_{a b}$. The present work shows the irrelevance of this second-order based interpretation and identifies alternative mechanisms. Section II describes a series of $\mathrm{Cu}$ (II) dimers, either fragments of magnetic lattices or molecular systems, on which calculations are performed. Sections III and IV situate the problem and recall the previous second-order based interpretations. In Sec. V truncated CI calculations are employed to show that the second-order corrections brought by the $2 h-1 p$ and $1 h-2 p$ excited configurations are in general much smaller than their contribution in the DDCI calculations. To get insight in the role of these excitations, a series of class-partitioned variational calculations is performed by adding selected classes of excited configurations to the valence CAS. The set of results confirms that the effect of $2 h-1 p$ and $1 h-2 p$ excitations is not a second-order one. In Sec. VI we try to identify which classes of excitations interact with them giving significant higherorder contributions to the magnetic coupling constant. Some general conclusions are given in Sec. VII.

\section{CHOICE OF TEST SYSTEMS AND COMPUTATIONAL DETAILS}

The systems considered here, represented in Fig. 1, contain two $\mathrm{Cu} d^{9}$ centers and differ by the number and type of bridging ligands and by the amplitude and sign of the magnetic constant. Geometries from x-ray crystal structures have been used for all the systems considered. A variety of complexes is reported with the following:

(a) one single bridging ligand, as in $\mathrm{La}_{2} \mathrm{CuO}_{4}$ (Refs. 35-38), and $\mathrm{Sr}_{2} \mathrm{CuO}_{2} \mathrm{Cl}_{2}$ (Ref. 39) cuprates, structures 1 and 2, respectively where a $\mathrm{Cu}_{2} \mathrm{O}_{7}$ fragment embed- 

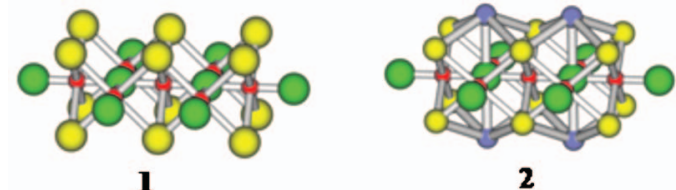

2

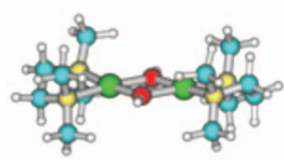

3

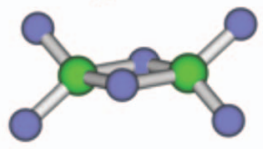

5

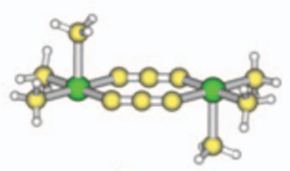

7

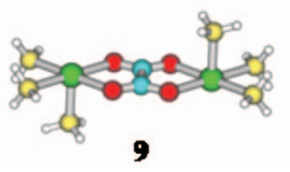

FIG. 1. Structures of binuclear Cu(II) compounds described in Sec. II.

ded in a set of point charges and total ion potentials simulating the Madelung potential of the infinite crystal has been chosen to evaluate the magnetic coupling;

(b) two $\mathrm{OH}^{-}$bridging ligands in the $\left[\mathrm{LCu}-(\mathrm{OH})_{2}-\mathrm{CuL}\right]$ complexes, giving AF coupling in system 3 (Refs. 40 and 41) with $\mathrm{L}=N, N, N^{\prime}, N^{\prime}$-tetramethylethylenediamine and $\mathrm{F}$ coupling in 4 (Refs. 42 and 43) with $\mathrm{L}=2^{\prime}$-bipyridine;

(c) two $\mathrm{Cl}^{-}$bridging ligands, giving also $\mathrm{F}$ coupling in the nonplanar $\left[\mathrm{Cu}-(\mathrm{Cl})_{2}-\mathrm{Cu}\right]$ unit in $\left[\mathrm{As}\left(\mathrm{C}_{6} \mathrm{H}_{5}\right)_{4}\right]$ $\times\left[\mathrm{CuCl}_{3}\right]$ (Refs. 44-46) and AF coupling for the planar geometry in $\mathrm{KCuCl}_{3}$, structures $\mathbf{5}$ and $\mathbf{6}$, respectively; ${ }^{44,47,48}$

(d) two bidentated end-to-end azido groups in $\left[\mathrm{LCu}-(\mathrm{NNN})_{2}-\mathrm{CuL}\right], \quad$ where $\mathrm{L}=N, N^{\prime}, N^{\prime \prime}$-trimethyl-1,4,7-triazacyclononane ${ }^{25,49}$ in structure 7 and

(e) one bisbidentated bridging ligand such as oxalato group in $\left[\mathrm{LCu}-\left(\mathrm{C}_{2} \mathrm{O}_{4}\right)-\mathrm{CuL}\right]$ with a large range of $\mathrm{AF}$ coupling constants for $\mathrm{L}=1,1,4,7,7$-pentaethyldiethylene-triamine in $\mathbf{8}^{50} \mathbf{9}$ is a model geometry of $\mathbf{8}$ used in previous works. ${ }^{51}$

In structures $\mathbf{7}$ and $\mathbf{8}$, external ligands were modeled with $\mathrm{NH}_{3}$ groups with the coordinated $\mathrm{N}$ at the experimental position. The counterions were not included in structures 3 and 5-8, since their distance to the anions was large.

In structures 1-7, core electrons of $\mathrm{Cu}$ atoms (up to $3 s$ ) were replaced with effective core potentials, and the $(9 s 6 p 6 d) /[3 s 3 p 4 d]$ basis set was used for the valence electrons of $\mathrm{Cu} .{ }^{52}$ For systems $\mathbf{8}$ and $\mathbf{9}, \mathrm{Cu}$ atoms are represented by means of atomic natural orbital ANO-type basis functions with contractions $[5 s 4 p 3 d 1 f]$ on the basis of our previous experience with these systems. ${ }^{21}$ For systems $\mathbf{1}$ and 2, O atoms basis set is of DZP (double zeta with polarization) quality with contractions $(10 s 5 p 1 d) /[3 s 2 p 1 d]$ as in previous works. $^{23}$ For the remaining systems, ANO-type basis functions are used for the ligands of different quality depending on the specific position. Contractions $[3 s 2 p 1 d]$ are employed for $\mathrm{C}, \mathrm{N}$, and $\mathrm{O}$ in bridging positions and $[4 s 3 p 1 d]$ for bridging $\mathrm{Cl}$. For the external ligands, contractions $[3 s 2 p]$ are used for atoms directly bonded to the metal centers and $[2 s 1 p]$ for the remaining positions. $\mathrm{H}$ atoms are represented by means of ANO-type functions with a contraction $[2 s 1 p]$ when placed on the bridging ligands, and $2 s$ or $1 s$ for the rest. ${ }^{53-55}$

At the variational level, the DDCI method has been used in its CASDI implementation. ${ }^{56}$ To analyze the effect of the different types of excitations, different truncated CI calculations have been performed including different subsets of excitation classes. Regarding the perturbative approaches, CASPT2 calculations have been performed from the minimal $(2,2)$ valence CAS by using the standard $H_{0}$ Hamiltonian implemented in MOLCAS 6.4 code. $^{57}$ These calculations use a different molecular orbital (MO) set for every state and are state specific (SS). The NEVPT2 method which uses a partially bielectronic $\hat{H}_{0}$ that avoids intruder states problem has been used in the partially contracted version. ${ }^{58-61}$ A direct evaluation of the second-order effect of $2 h-1 p$ and $1 h-2 p$ has also been coded independently, as will be commented in Sec. V A.

\section{IMPLICATIONS OF THE VARIATIONAL DEFINITION OF THE ORBITALS}

An initial crucial point is to precise how the magnetic orbitals are defined, since the use of mean-field variational calculations has specific consequences linked to Brillouin's theorem. ${ }^{62,63}$

Let us consider the basic problem of magnetic coupling between two $S=\frac{1}{2}$ spins located on two metallic sites $A$ and $B$, such as two $\mathrm{Cu} d^{9}$ ions. In a zeroth-order picture two unpaired electrons occupy two metal-centered orthogonal magnetic orbitals $a$ and $b$. These two electrons and two orbitals lead to one triplet and three singlet valence states. The magnetic orbitals may be uniquely defined through a restricted open-shell self-consistent field (SCF) calculation of the single determinant $T_{u}$ triplet state. The core closed-shell orbital $h$ is optimized as well. The $M s=0$ component of this $T_{u}$ state is

$$
T_{u}=\frac{1}{\sqrt{2}}(|h \bar{h} g \bar{u}|-|h \bar{h} u \bar{g}|)=\frac{1}{\sqrt{2}}(|h \bar{h} a \bar{b}|-|h \bar{h} b \bar{a}|),
$$

where $g$ and $u$ are symmetry adapted magnetic orbitals, and the localized $a$ and $b$ orbitals are obtained by rotation $a, b=1 / \sqrt{2}(g \pm u)$. Local orthogonal valence orbitals are the basic stones of a fruitful mode of analysis of the wave function and of the physics that it obeys, which has been a usual tool of interpretation of magnetic coupling ${ }^{8,33}$ and electron transfer processes ${ }^{64}$ (for recent contributions on orthogonal VB analysis of CAS wave functions, see Refs. 65 and 66).

The lowest singlet state is dominated by the neutral configuration $S_{N}$, 
TABLE I. Calculated magnetic coupling constants $\left(\mathrm{cm}^{-1}\right)$ for different partially truncated post-CASCI levels for systems 1-9.

\begin{tabular}{|c|c|c|c|c|c|c|c|c|c|}
\hline & $\begin{array}{c}\mathrm{La}_{2} \mathrm{CuO}_{4} \\
\mathbf{1}\end{array}$ & $\underset{2}{\mathrm{Sr}_{2} \mathrm{CuO}_{2} \mathrm{Cl}_{2}}$ & $\begin{array}{c}{\left[\mathrm{Cu}_{2}(\mathrm{OH})_{2}\right]} \\
\mathbf{3}\end{array}$ & $\begin{array}{c}{\left[\mathrm{Cu}_{2}(\mathrm{OH})_{2}\right]} \\
\mathbf{4}\end{array}$ & $\begin{array}{c}{\left[\mathrm{Cu}_{2} \mathrm{Cl}_{2}\right]} \\
\mathbf{5}\end{array}$ & $\begin{array}{c}{\left[\mathrm{Cu}_{2} \mathrm{Cl}_{2}\right]} \\
\quad 6\end{array}$ & $\begin{array}{c}{\left[\mathrm{Cu}_{2}\left(\mathrm{~N}_{3}\right)_{2}\right]} \\
7\end{array}$ & $\begin{array}{c}{\left[\mathrm{Cu}_{2}\left(\mathrm{C}_{2} \mathrm{O}_{4}\right)\right]} \\
\mathbf{8}\end{array}$ & $\begin{array}{c}{\left[\mathrm{Cu}_{2}\left(\mathrm{C}_{2} \mathrm{O}_{4}\right)\right]} \\
9\end{array}$ \\
\hline CAS & -255 & -160 & -35 & 33 & 13 & 11 & -82 & -7 & -13 \\
\hline $\mathrm{CAS}+\mathrm{S}$ & -706 & -464 & -159 & 73 & 28 & 17 & -362 & -22 & -39 \\
\hline DDCI2 & -744 & -482 & -184 & 63 & 24 & 8 & -375 & -23 & -41 \\
\hline DDCI $2+2 h-1 p$ & -1462 & -1256 & -750 & 195 & 80 & -61 & -1470 & -158 & -344 \\
\hline $\mathrm{DDCI} 2+1 h-2 p$ & -569 & -386 & -117 & 57 & 20 & 16 & -324 & -18 & -32 \\
\hline DDCI & -1077 & -952 & -500 & 157 & 54 & -21 & -802 & -78 & -160 \\
\hline Expt. & {$[-1030,-1096]^{\mathrm{a}}$} & $-1008^{\mathrm{b}}$ & $-509^{\mathrm{c}}$ & $172^{\mathrm{d}}$ & $46^{\mathrm{e}}$ & $0,-40^{\mathrm{e}}$ & $<-800^{\mathrm{f}}$ & $-75^{\mathrm{g}}$ & $\ldots$ \\
\hline
\end{tabular}

${ }^{\mathrm{a}}$ References 35-38.

${ }^{\mathrm{b}}$ Reference 39.

${ }^{\mathrm{c}}$ Reference 41.

${ }^{\mathrm{d}}$ Reference 43.

$$
\begin{aligned}
S_{g} & =\alpha|g \bar{g}|-\beta|u \bar{u}| \\
& =\lambda\left(\frac{|a \bar{b}|+|b \bar{a}|}{\sqrt{2}}\right)+\mu\left(\frac{|a \bar{a}|+|b \bar{b}|}{\sqrt{2}}\right)=\lambda S_{N}+\mu S_{I}, \\
\alpha & >\beta>0, \quad \lambda=\frac{\alpha+\beta}{\sqrt{2}} \gg \mu=\frac{\alpha-\beta}{\sqrt{2}} .
\end{aligned}
$$

As previously discussed, ${ }^{33}$ Brillouin's theorem that cancels the interaction between the triplet configuration and those obtained by single excitations also gives a negligible (for $1 \mathrm{~h}$ and $1 p$ ) or null (for $1 h-1 p$ ) interaction between the neutral singlet configuration $S_{N}$ and the singly excited ones.

Regarding the ionic components of the singlet state $I=\{|h \bar{h} a \bar{a}|,|h \bar{h} b \bar{b}|\}$, the interaction with $1 h$ (LMCT) configurations gives

$$
\left\langle h \bar{h} b \bar{b}|\hat{H}| \frac{1}{\sqrt{2}}\left(\hat{a}_{a}^{+} \hat{a}_{h}+\hat{a}_{\bar{a}}^{+} \hat{a}_{h}^{-}\right) h \bar{h} b \bar{b}\right\rangle=\sqrt{2}\left\langle h\left|\hat{J}_{b}-\hat{J}_{a}-\hat{K}_{b}\right| a\right\rangle .
$$

This interaction has a non-negligible AF effect due to $\left\langle h\left|\hat{J}_{a}\right| a\right\rangle$ term. Similar considerations apply for $1 p$ (MLCT) configurations. These non-negligible interactions will play a role in the forthcoming discussion.

The singlet $1 h-1 p$ excitations on the $I$ configurations lead to a strong interaction, reflecting the change in the electrostatic field with respect to the mean one,

$$
\begin{aligned}
\left\langle h \bar{h} b \bar{b}|\hat{H}| \frac{1}{\sqrt{2}}\left(\hat{a}_{p}^{+} \hat{a}_{h}+\hat{a}_{\bar{p}}^{+} \hat{a}_{\bar{h}}^{-}\right) h \bar{h} b \bar{b}\right\rangle \\
=\sqrt{2}\left\langle h\left|\hat{J}_{b}-\hat{J}_{a}+\frac{1}{2}\left(\hat{K}_{a}-\hat{K}_{b}\right)\right| p\right\rangle .
\end{aligned}
$$

If alternatively CASSCF singlet MOs are used, where the singlet state $S_{g}=\lambda S_{N}+\mu S_{I}$ has a small contribution of ionic VB components [see Eq. (3)], the magnetic orbitals are not strictly equivalent to those resulting from a SCF calculation of the triplet state, since they have larger tails on the ligands, making larger their interaction. The same argument holds for $1 p$ excitations.

A third possibility consists of using a different MO set for each state (SS calculations). This is the procedure, for
${ }^{\mathrm{e}}$ Reference 44.

${ }^{\mathrm{f}}$ Reference 49.

${ }^{\mathrm{g}}$ Reference 50

instance, in CASPT2 calculations. The use of SS orbitals in DDCI calculations makes null the differential contributions of the $1 h$ and $1 p$ excitation classes for both states because of Brillouin's theorem.

Although DDCI calculations are almost independent on the choice of the MO set and the difference between SCF triplet and CASSCF singlet MOs is not appreciable in the drawing of their amplitudes, the small differences have nevertheless consequences on the effect of the different types of excitations on $J$, as will be discussed in Sec. V A.

Whatever the SCF procedure for obtaining the MO set, the energy optimization produces an optimal (or nearly optimal) mixing of the metal and ligand orbitals. This has the crucial consequence of drastically reducing the weight of both the LMCT and the MLCT configurations, which belong to the $1 h$ and $1 p$ classes, respectively. Consequently, with a self-consistent MO set, the Anderson two-band model $^{2}$ cannot be the reference to understand the different contributions to the coupling, since in this case the hopping integrals between magnetic and ligand MOs, $t_{a l}$ and $t_{b l}$, are null. Variational optimization of the MOs compels one to stay in the simple one-band model.

\section{SECOND-ORDER EVALUATION OF THE MAGNETIC COUPLING}

At all stages of calculation the wave functions of the lowest triplet and singlet states are largely dominated by the neutral VB configurations $T_{u}$ and $S_{N}$, respectively, which may be considered as reference zeroth-order functions. Their energy difference is positive,

$$
\left\langle S_{N}|\hat{H}| S_{N}\right\rangle-\left\langle T_{u}|\hat{H}| T_{u}\right\rangle=2 K_{a b} .
$$

The interaction between the ionic and neutral determinants contributes to the second order to an energy stabilization of the singlet state $\varepsilon_{g}^{(2)}=-4 t_{a b}^{2} / U$, as derived in the one-band Anderson mechanism (1).

Numerical results have shown that in general the CASCI evaluation of $J$ gives at most $20 \%-30 \%$ of the experimental AF coupling constants, as illustrated by CAS entry in Table I, which reports the magnetic coupling values obtained with different $\mathrm{CI}$ spaces for the binuclear $\mathrm{Cu}$ (II) complexes described in Sec II. It is therefore necessary to go beyond va- 
lence configurations and to consider nonvalence configurations to reproduce the experimental values. As shown in the 1960s (Ref. 13) and numerically exploited in the 1980s on copper acetate ${ }^{8}$ and other $S=\frac{1}{2}$ dimers, ${ }^{9-12}$ only those determinants contributing at second order to the singlet-triplet energy difference must in principle be taken into account. When considering neutral determinants as zeroth-order space this differential list involves the valence ionic configurations as well as $1 h, 1 p, 2 h, 2 p$, and $1 h-1 p$ classes of excited determinants. This is the so-called DDCI2 space. The commonly used $\mathrm{CAS}+$ Singles $\mathrm{CI}(\mathrm{CAS}+\mathrm{S})$ only includes $1 h$, $1 p$, and $1 h-1 p$ classes. The CI restricted to this space usually gives $50 \%-60 \%$ of the experimental AF coupling, as shown in Table I (entries CAS $+\mathrm{S}$ and DDCI2). As discussed in Sec. III, the $1 h$ and $1 p$ configurations interact with the ionic VB component of the CAS wave function. Since the coefficient of this ionic configuration is small at this stage, the direct contributions of $1 h$ and $1 p$ on the singlet remain small. At these CI levels, the $1 h-1 p$ configurations play the leading role. Their effect may be split into the following:

(a) The spin polarization correction resulting from interaction of the neutral $T_{u}$ and $S_{N}$ configurations with the product of a triplet single excitation of the core electrons by a triplet configuration of the magnetic electrons of the type

$$
\hat{a}_{\bar{p}}^{+} \hat{a}_{b}^{+} \hat{a}_{b}^{-} \hat{a}_{h}|h \bar{h} a \bar{b}|=|\bar{h} \bar{p} a b| \text {. }
$$

This contribution is a second-order effect on the neutral zeroth-order functions $T_{u}$ and $S_{N}$ and may be either $\mathrm{F}$ or AF.

(b) The dynamical polarization of the ionic VB configurations resulting from the product of a singlet single excitation of the core electrons by a singlet single excitation of the magnetic electrons which can be written as a single excitation on the top of the ionic forms as:

$$
\frac{1}{\sqrt{2}}\left(\hat{a}_{p}^{+} \hat{a}_{h}+\hat{a}_{\bar{p}}^{+} \hat{a}_{h}^{-}\right)|h \bar{h} a \bar{a}|=\frac{1}{\sqrt{2}}(|p \bar{h} a \bar{a}|+|h \bar{p} a \bar{a}|) .
$$

The direct interaction of these configurations with the ionic valence ones is large and may be considered as lowering of the effective energy of ionic configurations

$$
U_{\mathrm{eff}}=U+\sum_{h, p} \frac{\langle h \bar{h} a \bar{a}|\hat{H}| p \bar{h} a \bar{a}\rangle\langle p \bar{h} a \bar{a}|\hat{H}| h \bar{h} a \bar{a}\rangle}{\left(-U+\varepsilon_{h}-\varepsilon_{p}\right)},
$$

where the matrix elements are given in Eq. (5).

As previously indicated, DDCI2 calculations include all the differential contributions to the energy difference between singlet and triplet states up to second order from a neutral reference space. The ionic valence configurations belong to these differential contributions but in view of their important role, it is preferable to include them in the reference space and to consider the CAS as a better reference space than the neutral VB determinants only. It increases the list of excitations, which have a differential effect on the energies of the valence states up to the second order, and the truncated CI space has to be enlarged as well. At this level, the purely inactive double excitations $\hat{a}_{p^{\prime}}^{+} \hat{a}_{p}^{+} \hat{a}_{h^{\prime}} \hat{a}_{h}$ induce an equal energy shift on the valence states and can be discarded. The so-obtained expansion is called the DDCI space, where $2 h-1 p$ and $1 h-2 p$ configurations are added to the DDCI2 one. Many calculations have shown the performance of DDCI estimates of the magnetic coupling. ${ }^{20-32}$ The results reported in Table I show that the $J$ values obtained at $\mathrm{CAS}+\mathrm{S}$ and DDCI2 levels are far from experimental ones, which suggest that an important part of the effects is missed. It is therefore concluded that $2 h-1 p$ and $1 h-2 p$ configurations bring from $30 \%$ to $50 \%$ of magnetic coupling. In the past these effects have been explained by suggesting that these excitations strengthen the coupling between the neutral and ionic forms $(N \leftrightarrow 2 h-1 p \leftrightarrow I)$, which produces an increase in $t_{a b}$ and consequently of the AF contribution of the magnetic coupling. The second-order correction to the effective hopping integral between neutral and ionic states mediated by the $2 h-1 p$ determinants is

$$
t_{a b}^{\mathrm{eff}}=t_{a b}+\frac{\left\langle S_{N}|\hat{H}| 2 h-1 p\right\rangle\langle 2 h-1 p|\hat{H}| I\rangle}{\Delta E_{2 h 1 p}}=t_{a b}+\Delta t_{a b}^{2 h 1 p}
$$

where the main contribution to $\Delta t_{a b}^{2 h 1 p}$ is

$$
\Delta t_{a b}^{2 h 1 p} \leftarrow-\sum_{h} \sum_{h^{\prime}} \sum_{p} \frac{2\left(h p, a h^{\prime}\right)\left(b h^{\prime}, h p\right)}{\Delta E_{2 h 1 p}} .
$$

The sign of $t_{a b}$ depends on the type of magnetic orbitals and is found negative in this type of systems. The sign of $\Delta t_{a b}^{2 h 1 p}$ depends on the symmetry of the inactive occupied $h^{\prime}$ orbital. It has been shown that if the contribution is dominated by single excitations on the LMCT configuration from antisymmetric orbital of the bridging ligand, as bridging oxygen $2 p_{z}$ orbital in cuprates, this contribution is negative and therefore increases its absolute value.

The $1 h-2 \mathrm{p}$ excitations also participate in the coupling between the ionic and neutral forms $(N \leftrightarrow 1 h-2 p \leftrightarrow I)$, modulating the effective hopping integral $t_{a b}$. In this case, the dominant contribution is

$$
\Delta t_{a b}^{1 h 2 p} \leftarrow \sum_{h} \sum_{p} \sum_{p^{\prime}} \frac{2\left(h p, a p^{\prime}\right)\left(b p^{\prime}, h p\right)}{\Delta E_{1 h 2 p}} .
$$

Similarly, the sign of these contributions also depends on the symmetry of $p^{\prime}$.

In the past, it has been assumed that the sum of these mechanisms results in an overall increase in $\left|t_{a b}^{\text {eff }}\right|$ and therefore of the kinetic exchange. Regarding the singlet and triplet wave functions, going from DDCI2 to DDCI produces a remarkable increase in the weights of the LMCT configurations, which has been related to the impact of the $2 h-1 p$ excitations. $^{23}$ In fact, single excitations on the LMCT configurations belong to the $2 h-1 p$ class of excitations and the effect of this excitation class was first interpreted as a lowering of the effective energy of the LMCT configurations. This would be relevant in a two-band model but since the LMCT configurations have a negligible interaction with the neutral determinants, this interpretation was discarded. On 
TABLE II. Effect of the MO set (T: SCF triplet MOs, S: CASSCF singlet MOs, SS: state specific MOs) on the magnetic coupling constant value $J$ (in $\mathrm{cm}^{-1}$ ) for systems 1-4 at different CI levels.

\begin{tabular}{|c|c|c|c|c|c|c|c|c|c|c|c|c|}
\hline \multirow{2}{*}{$\frac{J_{\text {expt }}}{\text { CI space }}$} & \multicolumn{3}{|c|}{$\begin{array}{c}\mathrm{La}_{2} \mathrm{CuO}_{4} \\
\mathbf{1} \\
{[-1030,-1096]^{\mathrm{a}}}\end{array}$} & \multicolumn{3}{|c|}{$\begin{array}{c}\mathrm{Sr}_{2} \mathrm{CuO}_{2} \mathrm{Cl}_{2} \\
\mathbf{2} \\
-1008^{\mathrm{b}}\end{array}$} & \multicolumn{3}{|c|}{$\begin{array}{c}{\left[\mathrm{Cu}_{2}(\mathrm{OH})_{2}\right]} \\
\mathbf{3} \\
-509^{\mathrm{c}}\end{array}$} & \multicolumn{3}{|c|}{$\begin{array}{c}{\left[\mathrm{Cu}_{2}(\mathrm{OH})_{2}\right]} \\
\mathbf{4} \\
+172^{\mathrm{d}}\end{array}$} \\
\hline & $\mathrm{T}$ & S & SS & $\mathrm{T}$ & $S$ & SS & $\mathrm{T}$ & $S$ & SS & $\mathrm{T}$ & $S$ & SS \\
\hline CAS & -255 & -387 & -315 & -160 & -237 & -195 & -35 & -72 & -52 & 33 & 31 & 32 \\
\hline $\mathrm{CAS}+\mathrm{S}$ & -706 & -780 & -783 & -464 & -505 & -507 & -159 & -178 & -179 & 73 & 73 & 74 \\
\hline DDCI2 & -744 & -825 & -828 & -482 & -527 & -530 & -184 & -205 & -206 & 63 & 62 & 64 \\
\hline DDCI & -1077 & -1149 & -1174 & -952 & -1002 & -1026 & -500 & -523 & -526 & 157 & 156 & 178 \\
\hline
\end{tabular}

${ }^{\mathrm{a}}$ References 35-38.

${ }^{\mathrm{b}}$ Reference 39.

the basis of this remark, two pathways were proposed to explain the enhancement of AF character as follows:

(1) A third-order mechanism, $N \leftrightarrow I \leftrightarrow(2 h-1 p) \leftrightarrow N$, which involves $\Delta t_{a b}$.

(2) A fourth-order one, $N \leftrightarrow(2 h-1 p) \leftrightarrow I \leftrightarrow(2 h-1 p) \leftrightarrow N$, which involves $\left|\Delta t_{a b}\right|^{2}$.

\section{IRRELEVANCE OF THE SECOND-ORDER INTERPRETATION OF THE ROLE OF THE $2 \boldsymbol{h}-1 \boldsymbol{p}$ AND $1 h-2 p$ EXCITED CONFIGURATIONS}

On the basis of the perturbative interpretation one may be tempted to rely on CAS-based second-order calculations or to combine DDCI2 variational calculations with perturbative estimates of the effect of $2 h-1 p$ and $1 h-2 p$ configurations, which are the largest subspaces in DDCI method, thus saving most of the computational effort. Class-partitioned variational calculations which add separately classes of excited configurations to the CAS may alternatively be considered in order to check whether the effect of the various classes is essentially additive, as they should be if dominated by second-order effects.

For the sake of comparison with the perturbative methods (by default SS) and to check the behavior of DDCI results with respect to the MO set, coupling constants for systems 1-4 have been evaluated from different sets of MOs: SCF triplet MOs, $(2,2)$ CASSCF singlet MOs, as well as the SS MOs. Table II shows that DDCI results are remarkably independent of the set of MOs used. Hereafter, we will concentrate on compounds $\mathbf{1 - 4}$ to perform a detailed analysis.

\section{A. Second-order CAS-based perturbations}

For consistency of comparison with class-partitioned variational calculations, although CASPT2 calculations are in general based on enlarged CAS, ${ }^{24}$ only $(2,2)$ minimal CAS will be considered here. The same strategy is applied in the NEVPT2 calculations. In both methods, the energy corrections to singlet and triplet states are class additive and so is the energy difference.

Tables III and IV report the details of the contributions of different classes for compounds $\mathbf{1 - 4}$. The perturbative calculations suffer for some arbitrariness in the definition of the zeroth-order Hamiltonian. In order to avoid this source of uncertainty and to check whether the class contributions are
${ }^{\mathrm{c}}$ Reference 41.

${ }^{\mathrm{d}}$ Reference 43 .

additive, variational calculations have been performed by adding specific subspaces of the outer space determinants to the CAS. The CI spaces are thus composed of the CAS and the indicated set of determinants. To evaluate the independent effect of each contribution on $J$, the CAS $J$ value is subtracted. This makes the comparison with perturbative contributions possible.

From this series of class-partitioned calculations, some relevant observations can be made as follows:

(a) The $2 h-2 p$ inactive double excitations bring a negligible contribution, despite the fact that the MOs are different from the singlet and the triplet states. This validates their elimination in DDCI method.

(b) There is a good agreement between the CASPT2 and CI calculations from $1 h$ to $2 p$ differential contributions. When the CI space includes all these contributions (DDCI2 level), the total result of $J$ is similar, which indicates the approximately additive character of all these excitation subsets.

(c) $1 \mathrm{~h}$ and $1 p$ contributions, which are obviously null in SS calculations, have a small AF effect when using a unique set of MOs in the variational calculations, as discussed in Sec. III.

(d) The overall CASPT2 $J$ value is very good in $\mathrm{La}_{2} \mathrm{CuO}_{4}$ 1 but significantly underestimated for $\mathrm{Sr}_{2} \mathrm{CuO}_{2} \mathrm{Cl}_{2} 2$ for the AF complex $\left[\mathrm{Cu}_{2}(\mathrm{OH})_{2}\right] \mathbf{3}$, as well as for the $\mathrm{F}$ complex $\left[\mathrm{Cu}_{2}(\mathrm{OH})_{2}\right] 4(66 \%, 50 \%$, and $50 \%$ of the experimental value, respectively). The NEVPT2 values are even smaller than the CASPT2 ones with the same distribution between the various classes. The relative failure of the second-order approaches already reported in previous works ${ }^{28,67}$ might possibly be attributed to the internally contracted character of these formalisms. The zeroth-order wave function severely underestimates the coefficient of the ionic VB component of the singlet state. The dynamical polarization effects included in $1 h-1 p$ excitations increase this coefficient, as recalled in Sec. IV. We have therefore tested the performance of a recently proposed procedure which allows a decontraction of the CAS composition of the wave function. ${ }^{68}$ This procedure actually leads to an enlargement of the ionic component resulting in an increase in the AF character. The results are excellent for system 1 since this procedure gives $J=-1106 \mathrm{~cm}^{-1}$ 
TABLE III. Independent contributions of various classes of excitations in perturbative and variational approaches for systems $\mathbf{1}$ and $\mathbf{2}$ (in $\mathrm{cm}^{-1}$ ). The truncated CI spaces include the corresponding excitation class on top of the CAS.

\begin{tabular}{|c|c|c|c|c|}
\hline & \multicolumn{4}{|c|}{$\begin{array}{c}\mathrm{La}_{2} \mathrm{CuO}_{4} \\
\mathbf{1} \\
J_{\text {expt }}=[-1030,-1096]^{\mathrm{a}}\end{array}$} \\
\hline & CASPT2 SS MOs & NEVPT2 SS MOs & Truncated CI SS MOs & Truncated CI T MOs \\
\hline $\operatorname{CAS}(2,2)$ & -315 & -315 & -315 & -255 \\
\hline $1 h$ & 0 & 0 & 0 & -21 \\
\hline $1 p$ & 0 & 0 & 0 & -30 \\
\hline $1 h-1 p$ & -434 & -301 & -418 & -268 \\
\hline $2 h$ & -16 & -5 & & \\
\hline $2 p$ & -7 & -6 & & \\
\hline $1 h-2 p$ & 77 & 38 & 50 & 66 \\
\hline $2 h-1 p$ & -287 & -221 & -181 & -19 \\
\hline $2 h-2 p$ & 16 & 16 & & \\
\hline \multirow[t]{3}{*}{ Total } & -966 & -795 & $-1174^{\mathrm{b}}$ & $-1077^{b}$ \\
\hline & \multicolumn{4}{|c|}{$\begin{array}{c}\mathrm{Sr}_{2} \mathrm{CuO}_{2} \mathrm{Cl}_{2} \\
\mathbf{2} \\
J_{\text {expt }}=-1008^{\mathrm{c}}\end{array}$} \\
\hline & CASPT2 SS MOs & NEVPT2 SS MOs & Truncated CI SS MOs & Truncated CI T MOs \\
\hline $\operatorname{CAS}(2,2)$ & -195 & -195 & -195 & -160 \\
\hline $1 h$ & 0 & 0 & 0 & -9 \\
\hline $1 p$ & 0 & 0 & 0 & -20 \\
\hline $1 h-1 p$ & -294 & -190 & -269 & -176 \\
\hline $2 h$ & -9 & -2 & & \\
\hline $2 p$ & -3 & -3 & & \\
\hline $1 h-2 p$ & 40 & 16 & 22 & 37 \\
\hline $2 h-1 p$ & -230 & -164 & -137 & -7 \\
\hline $2 h-2 p$ & 25 & 23 & & \\
\hline Total & -667 & -515 & $-1008^{b}$ & $-952^{\mathrm{b}}$ \\
\hline
\end{tabular}

${ }^{\mathrm{a}}$ References 35-38.

${ }^{\mathrm{b}}$ This value is not the sum of independent class contributions but that of the DDCI calculation.

${ }^{\mathrm{c}}$ Reference 39.

with the NEVPT2 externally partially contracted version, instead of $-795 \mathrm{~cm}^{-1}$ before the internal decontraction, as reported in Table III. But they remain unsatisfactory for system 2, $J=-690 \mathrm{~cm}^{-1}$, after the decontraction instead of $-515 \mathrm{~cm}^{-1}$, far from the experiment, $-1008 \mathrm{~cm}^{-1}$, and DDCI, $-952 \mathrm{~cm}^{-1}$, and similarly for system $\mathbf{3}$, where the internal decontraction gives $J=-284 \mathrm{~cm}^{-1}$, a significant improvement when compared to $-173 \mathrm{~cm}^{-1}$ (see Table IV), but still insufficient since the experimental value is $-509 \mathrm{~cm}^{-1}$. Therefore the internal contraction cannot be considered the main responsible for the failure of the second-order treatments.

(e) The effect of $1 h-2 p$ excitations is similar in perturbative and class-partitioned CI calculations with a moderate $\mathrm{F}$ contribution to the $J$ value in AF systems $\mathbf{1 - 3}$, and an almost negligible AF contribution in F system 4.

(f) The direct effect of $2 h-1 p$ configurations both in perturbative and class-partitioned CI calculations deserves a more accurate analysis. This class of excitations does not bring a significant contribution to $J$ when a common set of MOs is used in the variational CAS
$+2 h-1 p$ calculations, as shown in the rightward columns of Tables III and IV $(-19,-7$, and -3 in compounds 1-3). This result questions the interpretation of their role given in Ref. 33 and recalled in Sec. IV. NEVPT2 calculations performed with triplet state MOs for both states give the confirmation: the contribution to $J$ of this class of excitations is small: $-45,-31$, -20 , and $3 \mathrm{~cm}^{-1}$ for $\mathbf{1 - 4}$, respectively. As a further verification, the second-order correction to $t_{a b}$ given in expressions (11) and (12) has been directly computed, and a negligible contribution has also been found. The second-order contribution observed in CASPT2 and NEVPT2 calculations in Tables III and IV is linked to the state specificity, as confirmed by SS variational calculations (column 3 in both tables). This effect can be understood as due to larger metal-ligand mixing of the magnetic orbitals in the singlet state, as discussed in Sec. III. The $\left(h p, a h^{\prime}\right),\left(h p, b h^{\prime}\right)$ integrals in expression (11) have larger amplitude for singlet state orbitals, since they are proportional to this mixing. It results in a larger stabilization of the singlet state and therefore in an enhancement of the AF character. An independent 
TABLE IV. Independent contributions of various classes of excitations in perturbative and variational approaches for systems $\mathbf{3}$ and $\mathbf{4}\left(\mathrm{in}_{\mathrm{cm}}^{-1}\right.$ ). The truncated CI spaces include the corresponding excitation class on top of the CAS.

\begin{tabular}{|c|c|c|c|c|}
\hline & \multicolumn{4}{|c|}{$\begin{array}{c}{\left[\mathrm{Cu}_{2}(\mathrm{OH})_{2}\right]} \\
\mathbf{3} \\
J_{\text {expt }}=-509^{\text {a }}\end{array}$} \\
\hline & CASPT2 SS MOs & NEVPT2 SS MOs & Truncated CI SS MOs & Truncated CI T MOs \\
\hline $\operatorname{CAS}(2,2)$ & -52 & -52 & -52 & -35 \\
\hline $1 h$ & 0 & 0 & 0 & -2 \\
\hline $1 p$ & 0 & 0 & 0 & -14 \\
\hline $1 h-1 p$ & -120 & -72 & -106 & -67 \\
\hline $2 h$ & -22 & -3 & & \\
\hline $2 p$ & -4 & -8 & & \\
\hline $1 h-2 p$ & 43 & 26 & 30 & 25 \\
\hline $2 h-1 p$ & -94 & -61 & -42 & -3 \\
\hline $2 h-2 p$ & -4 & -4 & & \\
\hline \multirow[t]{3}{*}{ Total } & -253 & -173 & $-526^{\mathrm{b}}$ & $-500^{\mathrm{b}}$ \\
\hline & \multicolumn{4}{|c|}{$\begin{array}{c}{\left[\mathrm{Cu}_{2}(\mathrm{OH})_{2}\right]} \\
\mathbf{4} \\
J_{\text {expt }}=+172^{\mathrm{c}}\end{array}$} \\
\hline & CASPT2 SS MOs & NEVPT2 SS MOs & Truncated CI SS MOs & Truncated CI T MOs \\
\hline $\operatorname{CAS}(2,2)$ & 32 & 32 & 32 & 33 \\
\hline $1 h$ & 0 & 0 & 0 & 0 \\
\hline $1 p$ & 0 & 0 & 0 & 1 \\
\hline $1 h-1 p$ & 22 & 21 & 36 & 28 \\
\hline $2 h$ & -13 & -2 & & \\
\hline $2 p$ & -3 & -5 & & \\
\hline $1 h-2 p$ & 15 & 18 & 22 & 3 \\
\hline $2 h-1 p$ & 62 & 50 & 60 & 7 \\
\hline $2 h-2 p$ & -24 & -20 & & \\
\hline Total & 95 & 94 & $178^{\mathrm{b}}$ & $157^{\mathrm{b}}$ \\
\hline
\end{tabular}

${ }^{\mathrm{a}}$ Reference 41.

${ }^{\mathrm{b}}$ This value is not the sum of independent class contributions but that of the DDCI calculation.

${ }^{\mathrm{c}}$ Reference 43.

calculation of $\Delta t_{a b}$ directly coded and evaluated confirms the SS character of the role of $2 h-1 p$ and $1 h-2 p$ classes in perturbative calculations. In any case, it must be noticed that the $2 h-1 p$ second-order correction in SS approaches is in general not sufficient and let the second-order calculation far from experiment.

(g) The most important evidence of this set of classpartitioned results is the very different impact of the $2 h-1 p$ and $1 h-2 p$ classes of excitations at the CI level when acting on the bare CAS or when added to the DDCI2 space. For compound 1, for instance, as reported in Table $\mathrm{V}, J$ at $\mathrm{CAS}+2 h-1 p$ level with triplet MOs is $-273 \mathrm{~cm}^{-1}$, i.e., the $2 h-1 p$ class gives an AF contribution of $-19 \mathrm{~cm}^{-1}$, while at DDCI $2+2 h-1 p J$ is $-1462 \mathrm{~cm}^{-1}$, i.e., an AF enhancing of $-718 \mathrm{~cm}^{-1}$ with respect to DDCI2 value. This value is largely overestimated when compared to experiment but when adding $1 h-2 p$ excitations (DDCI space), a correct value of $-1077 \mathrm{~cm}^{-1}$ is obtained due to the opposite effect of this last class of excitations. Similar conclusions can be extracted for systems $\mathbf{2} \mathbf{- 4}$.

From these observations it may be concluded that the large overall effect of these two classes, as it appears in the variational calculations going from DDCI2 to DDCI, is not a second-order effect. It proceeds through their interaction with other classes of nonvalence determinants. To elucidate the mechanism of their indirect impact on the $J$ values, truncated CI gathering various classes of excitations have been performed.

\section{B. Tracing the indirect mechanism of action of $2 h-1 p$ and $1 h-2 p$ excitations}

Table $\mathrm{V}$ reports the CI results on systems 1-4 when adding $2 h-1 p$ and/or $1 h-2 p$ excitations to different truncated CI spaces. In AF systems 1-3, the $2 h-1 p$ excitations only bring an important $\mathrm{AF}$ contribution once $1 h$ LMCT configurations have been added to the CAS. Considering 1 as an example, while the addition of $2 h-1 p$ to the CAS only brings a correction of $-19 \mathrm{~cm}^{-1}$ to $J$, an effect of $-240 \mathrm{~cm}^{-1}$ is obtained when the $1 h$ configurations are present in the CI space. The AF effect of this class of excitations is dramatically amplified when the $1 h-1 p$ configurations are included in the CI, this contribution being $-672 \mathrm{~cm}^{-1}$ in system $1(\mathrm{CAS}+\mathrm{S}$, $2 h-1 p$ entry in Table V). On the other hand, the $1 p$ excitations have a negligible effect. 
TABLE V. Effect of adding $2 h-1 p$ or $1 h-2 p$ classes of excitations to CAS, CAS $+\mathrm{S}$, and DDCI2 spaces (in $\mathrm{cm}^{-1}$ ) for systems 1-4 (T MOs). $\Delta J$ is referred to $\mathrm{CAS}, \mathrm{CAS}+\mathrm{S}$, or DDCI2 values, respectively.

\begin{tabular}{|c|c|c|c|c|c|c|c|c|}
\hline \multirow[t]{2}{*}{$J_{\text {expt }}$} & \multicolumn{2}{|c|}{$\begin{array}{c}\mathrm{La}_{2} \mathrm{CuO}_{4} \\
\mathbf{1} \\
{[-1030,-1096]^{\mathrm{a}}}\end{array}$} & \multicolumn{2}{|c|}{$\begin{array}{c}\mathrm{Sr}_{2} \mathrm{CuO}_{2} \mathrm{Cl}_{2} \\
\mathbf{2} \\
-1008^{\mathrm{b}} \\
\end{array}$} & \multicolumn{2}{|c|}{$\begin{array}{c}{\left[\mathrm{Cu}_{2}(\mathrm{OH})_{2}\right]} \\
\mathbf{3} \\
-509^{\mathrm{c}}\end{array}$} & \multicolumn{2}{|c|}{$\begin{array}{c}{\left[\mathrm{Cu}_{2}(\mathrm{OH})_{2}\right]} \\
\mathbf{4} \\
+172^{\mathrm{d}}\end{array}$} \\
\hline & $J$ & $\Delta J$ & $J$ & $\Delta J$ & $J$ & $\Delta J$ & $J$ & $\Delta J$ \\
\hline $\operatorname{CAS}(2,2)$ & -255 & $\cdots$ & -160 & $\cdots$ & -35 & $\cdots$ & 33 & $\cdots$ \\
\hline$+2 h-1 p$ & -273 & -18 & -167 & -7 & -38 & -3 & 40 & 7 \\
\hline$+1 h+2 h-1 p$ & -494 & -240 & -381 & -221 & -100 & -65 & 182 & 149 \\
\hline$+1 h-2 p$ & -195 & 66 & -123 & 37 & -10 & 25 & 36 & 3 \\
\hline$+1 p+1 h-2 p$ & -221 & 33 & -140 & 20 & -14 & 21 & 41 & 8 \\
\hline $\mathrm{CAS}+\mathrm{S}$ & -706 & $\cdots$ & -464 & $\cdots$ & -159 & $\cdots$ & 73 & $\cdots$ \\
\hline$+2 h-1 p$ & -1378 & -672 & -1199 & -735 & -650 & -523 & 248 & 175 \\
\hline$+1 h-2 p$ & -542 & 164 & -372 & 92 & -94 & 65 & 71 & -2 \\
\hline$+2 h-1 p+1 h-2 p$ & -1023 & -317 & -916 & -452 & -428 & -269 & 203 & 130 \\
\hline DDCI2 & -744 & & -482 & & -184 & & 63 & \\
\hline$+2 h-1 p$ & -1462 & -718 & -1256 & -774 & -750 & -566 & 195 & 132 \\
\hline$+1 h-2 p$ & -569 & 175 & -386 & 96 & -117 & 67 & 57 & -6 \\
\hline DDCI & -1077 & & -952 & & -500 & & 157 & \\
\hline DDCI-DDCI2 & & -333 & & -470 & & -316 & & 94 \\
\hline
\end{tabular}

${ }^{\mathrm{a}}$ References 35-38.

${ }^{\mathrm{b}}$ Reference 39.

\section{${ }^{c}$ Reference 41.}

${ }^{\mathrm{d}}$ Reference 43 .
The effect is similar but of opposite sign in the F system 4 , since $2 h-1 p$ excitations bring an important $\mathrm{F}$ contribution only when the $1 h$ LMCT configurations are present in the CI space, $149 \mathrm{~cm}^{-1}$. The dominant mechanisms of action of $2 h-1 p$ configurations must therefore be different in $\mathrm{AF}$ and $\mathrm{F}$ systems.

The effect of $1 h-2 p$ configurations is $\mathrm{F}$ in AF systems, while it is negligible in the F system $\mathbf{4}$. In systems $\mathbf{1 - 3}$ this $F$ effect increases when $1 h-2 p$ excitations are added to CI space from CAS to DDCI2: for $1, \Delta J=66 \mathrm{~cm}^{-1}$ for $\mathrm{CAS}+1 h-2 p$ space, $164 \mathrm{~cm}^{-1}$ for $\mathrm{CAS}+\mathrm{S}+1 h-2 p$, and $175 \mathrm{~cm}^{-1}$ for DDCI $2+1 h-2 p$. Since the same trend but larger in absolute value is observed for $2 h-1 p$ excitations, this $\mathrm{F}$ effect seems to be a damping of the AF mechanisms.

In Sec. VI, some rationalizations of these effects will be proposed.

\section{RATIONALIZATION OF THE ROLE OF $2 h-1 p$ AND $1 h-2 p$ EXCITATIONS}

\section{A. AF effect of $2 h-1 p$ configurations in AF systems}

It has previously been $\operatorname{shown}^{33}$ that the $2 h-1 p$ class of excitations is responsible for the occurrence of large coefficients of LMCT $(1 h)$ configurations. In that work the increase in the LMCT coefficients was not supposed to have an impact on the magnetic coupling constant, since the direct interaction between LMCT and the neutral configurations is negligible according to Brillouin's theorem, as discussed in Sec. III. However, there is an important interaction between LMCT and ionic configurations ruled by the magnitude of the $\left\langle h\left|\hat{J}_{a}\right| a\right\rangle$ term. One may therefore suspect that the interaction between LMCT and ionic configurations has a signifi-

TABLE VI. Some representative coefficients of the singlet wave function at different CI levels for systems $\mathbf{1}, \mathbf{3}$, and $\mathbf{4}$. $C_{I} / C_{N}$, ionic/neutral valence configurations ratio. LMCT, the most significant LMCT excitation in the singlet state. The left column indicates the specific excitation class(es) added in each case to CAS, CAS +S, or DDCI2 spaces.

\begin{tabular}{|c|c|c|c|c|c|c|c|c|c|}
\hline & \multicolumn{3}{|c|}{$\mathrm{La}_{2} \mathrm{CuO}_{4} \mathrm{I}$} & \multicolumn{3}{|c|}{$\left[\mathrm{Cu}_{2}(\mathrm{OH})_{2}\right] \mathbf{3}$} & \multicolumn{3}{|c|}{$\left[\mathrm{Cu}_{2}(\mathrm{OH})_{2}\right] \mathbf{4}$} \\
\hline & $J$ & $C_{I} / C_{N}$ & LMCT & $J$ & $C_{I} / C_{N}$ & LMCT & $J$ & $C_{I} / C_{N}$ & LMCT \\
\hline CAS & -255 & 0.0406 & $\ldots$ & -35 & 0.0218 & $\ldots$ & 33 & 0.0009 & $\cdots$ \\
\hline $1 h+2 h-1 p$ & -494 & 0.0685 & 0.1312 & -100 & 0.0495 & 0.1158 & 182 & 0.0060 & 0.1022 \\
\hline $1 p+1 h-2 p$ & -221 & 0.0406 & $<0.001$ & -14 & 0.0215 & $<0.001$ & 41 & 0.0010 & $<0.001$ \\
\hline $\mathrm{CAS}+\mathrm{S}$ & -706 & 0.0910 & 0.0292 & -159 & 0.0544 & 0.0197 & 73 & 0.0005 & 0.0098 \\
\hline $2 h-1 p$ & -1378 & 0.1385 & 0.1615 & -650 & 0.1159 & 0.1483 & 248 & 0.0173 & 0.1225 \\
\hline $1 h-2 p$ & -542 & 0.0789 & 0.0222 & -94 & 0.0459 & 0.0158 & 71 & 0.0017 & 0.0087 \\
\hline $2 h-1 p+1 h-2 p$ & -1023 & 0.1137 & 0.1239 & -428 & 0.0926 & 0.1197 & 203 & 0.0005 & 0.1017 \\
\hline DDCI2 & -744 & 0.0955 & 0.0300 & -184 & 0.0571 & 0.0208 & 63 & 0.0004 & 0.0104 \\
\hline $2 h-1 p$ & -1462 & 0.1452 & 0.1632 & -750 & 0.1218 & 0.1514 & 195 & 0.0181 & 0.1244 \\
\hline $1 h-2 p$ & -569 & 0.0818 & 0.0227 & -117 & 0.0478 & 0.0166 & 57 & 0.0017 & 0.0092 \\
\hline DDCI & -1077 & 0.1180 & 0.1250 & -500 & 0.0966 & 0.1218 & 157 & 0.0132 & 0.1031 \\
\hline
\end{tabular}


cant impact on the weight of the ionic valence configurations. Table VI reports the variation in the ionic/neutral valence coefficient ratio $C_{I} / C_{N}$ and of the largest LMCT (1h) coefficient in the singlet state with the composition of the CI space for systems $\mathbf{1}, \mathbf{3}$, and $\mathbf{4}$. For system $\mathbf{1}$, for instance, LMCT coefficient is small, 0.03, at CAS $+\mathrm{S}$ and DDCI2 levels but is dramatically enhanced, 0.16 , when adding $2 h-1 p$ at
$\mathrm{CAS}+\mathrm{S}$ or DDCI2 space, and damped to 0.12 when completing the DDCI space with $1 h-2 p$ excitations. The same considerations can be extended to other systems. The variation in the $C_{I} / C_{N}$ ratio follows the same trend.

The specific effect of $2 h-1 p$ configurations on the singlet state energy can be interpreted on the basis of the following mechanism:

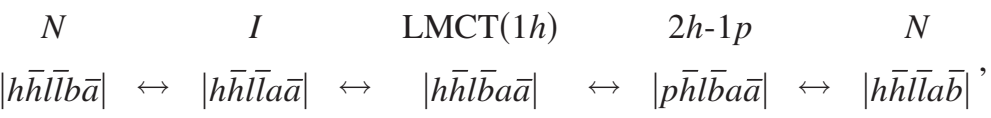

or the left-right counterpart, where $l$ is a ligand orbital. This mechanism is of third order with respect to the CAS and of fourth order with respect to the neutral determinants, and is represented in Diagram 1 where the vertical rectangles represent the deviations of the field with respect to the mean field.

From this mechanism, it can be understood that the component of the singlet wave function on the LMCT determinants is not essentially obtained through their interaction with the ionic valence configuration, and that a $2 h-1 p$ correlation effect contributes to the mixing between the metal and ligand orbitals in the natural magnetic orbitals, as observed in Ref. 34.
This mechanism involves CAS, $1 h$, and $2 h-1 p$ configurations. However a truncated $\mathrm{CI}$ calculation on this space only gives $50 \%$ of DDCI $J$ value, as shown in Table $\mathrm{V}$ in $\mathrm{CAS}+1 h+2 h 1 p$ entry. Some additional steps to this mechanism introduce the dynamical polarization of the intermediate state $I$ and/or LMCT, resulting in higher-order contributions. $1 h-1 p$ excitations on the top of the ionic configurations lower their effective energy, as do $1 h-1 p$ excitations on the top of LMCT configurations. Therefore, the observed increase in the impact on the $2 h-1 p \leftrightarrow \mathrm{LMCT}$ combined action with the $1 h-1 p$ configurations can be interpreted as a higherorder pathway,

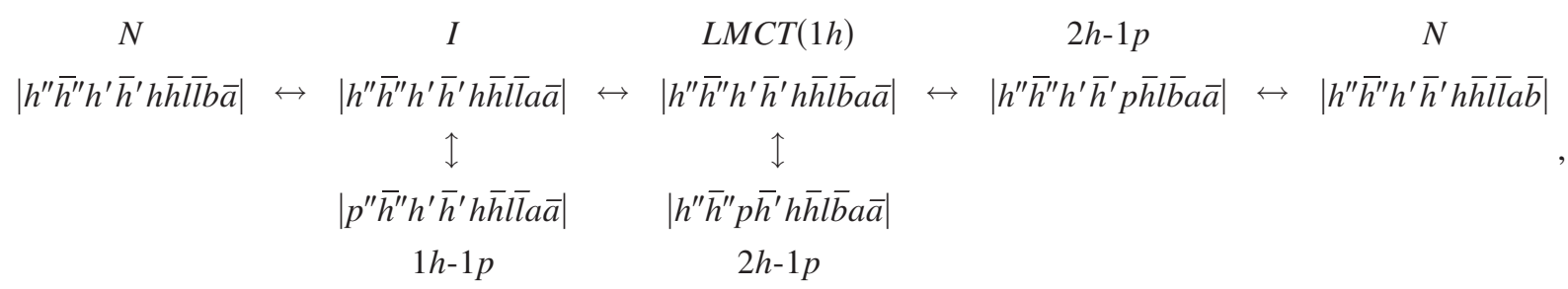

where $\left|h^{\prime \prime} \bar{h}^{\prime \prime} p^{\prime} \bar{h}^{\prime} h \bar{h} \bar{l} \bar{l} a\right|$ and/or $\left|p^{\prime \prime} \bar{h}^{\prime \prime} h^{\prime} \bar{h}^{\prime} h \bar{h} b \bar{l} a \bar{a}\right|$ determinants decrease the effective energy of $I$ and/or LMCT configurations of the precedent mechanism. Diagram 2 represents the inclusion of all these higher-order effects on Diagram 1. This proposal is supported by the results on Table $\mathrm{V}$, where $\mathrm{CAS}+\mathrm{S}+2 h 1 p$ that contains also $1 h-1 p$ excitations enhances considerably the AF character of $J$, although now slightly overestimated, as discussed in Sec. V.

This analysis in terms of fourth-order effects explains why internally contracted third-order perturbation theory is unable to improve the second-order values of $J$, as observed in NEVPT3 (Ref. 69) calculations performed on $\mathbf{3}$. The inclusion of the third-order correction gives a still more underestimated value of $J$ by $20 \mathrm{~cm}^{-1}$. The internal contraction prevents the revision of the CAS weight of the ionic configuration, as discussed in Sec. V A.

Finally, a somewhat simpler mechanism can also be considered,

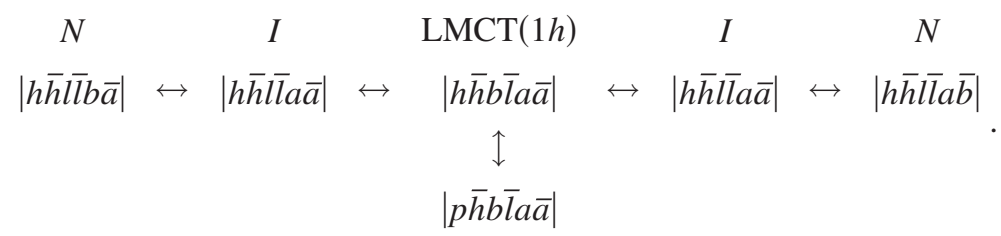




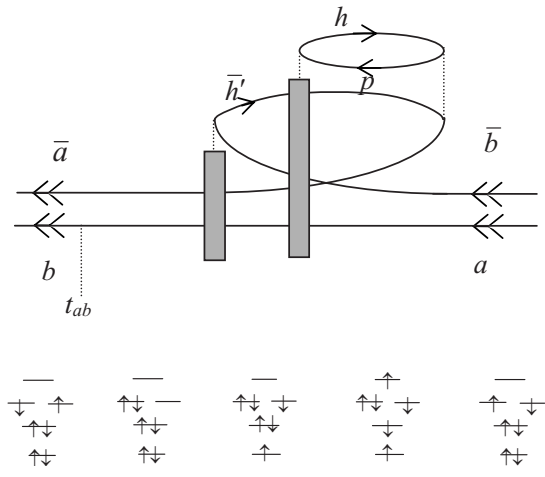

Diagram 1.

Since the parent mechanism $N \leftrightarrow I \leftrightarrow \operatorname{LMCT}(1 h) \leftrightarrow I \leftrightarrow N$ plays a negligible role, it is not expected to play any important role.

\section{B. Ferromagnetic role of $2 h-1 p$ configurations in $\mathrm{F}$ systems}

The above mechanisms are based on the interaction mediated by the ionic configurations, which do not play any role in $\mathrm{F}$ systems since they have practically null coefficients in the singlet wave function (see $C_{I} / C_{N}$ ratio for compound 4 in Table VI). The differential effect of $2 h-1 p$ excitations on singlet and triplet states must therefore be different from the preceding ones. This is corroborated by the opposite sign of the effect of $2 h-1 p$ excitations in F system 4 (Tables III-V). This suggests that instead of the ionic configurations, some other intermediates play a role. Among the possible interactions, a simple mechanism involving the same set of excitations may be

$$
N \leftrightarrow 2 h-1 p \leftrightarrow \operatorname{LMCT}(1 h) \leftrightarrow 2 h-1 p \leftrightarrow N .
$$

The diagrammatic transcription of this mechanism is represented in Diagram 3.

In this mechanism, the intermediate state LMCT is of lower energy for the triplet state than for the singlet. The LMCT triplet $1 / \sqrt{2}|(b \bar{h}-h \bar{b}) a \bar{a}\rangle$ is more stable than the LMCT singlet $1 / \sqrt{2}|(b \bar{h}+h \bar{b}) a \bar{a}\rangle$ by $2 K_{h b}$. The integral $K_{h b}$ is

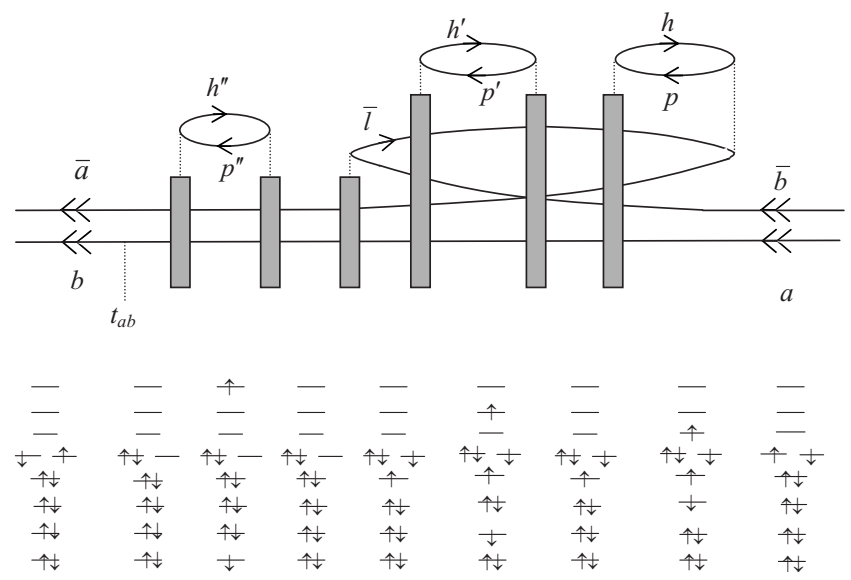

Diagram 2.
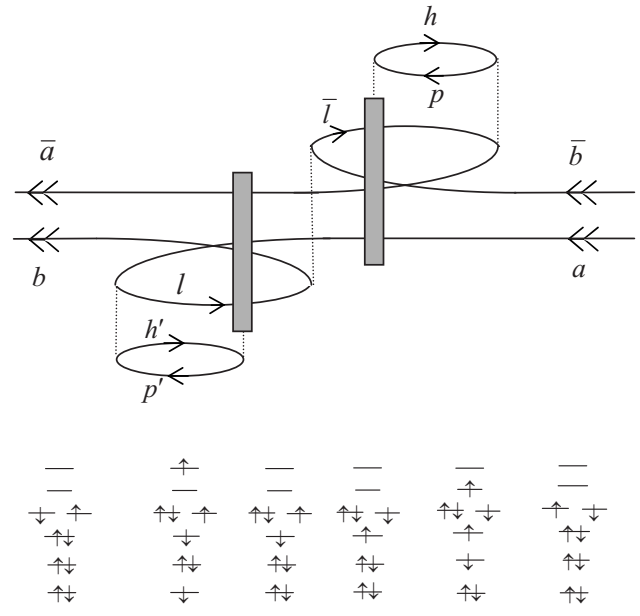

Diagram 3.

far from being negligible. Since the energy of the intermediate state is lower for the triplet state, it will act more significantly on this state.

Notice that this last mechanism also works ferromagnetically on AF systems. There are therefore several competitive pathways playing in favor of AF or $\mathrm{F}$ contributions which become dominant depending on the weight of the ionic configurations and/or the relative energy of intermediate configurations.

An alternative analysis of the consequences of the precedent mechanism may be done in terms of the effect on the natural orbitals obtained after the CI. The increase in the weight of the LMCT component due to the effect of $2 h-1 p$ excitations increases the ligand-metal mixing. This effect is observed in the natural magnetic orbitals obtained at the DDCI level, as extensively discussed previously. ${ }^{70}$ This delocalization of the magnetic orbitals results in both an increase in the effective hopping integral $t_{a b}$ and of the direct exchange integral $K_{a b}$. Depending on the prevailing effect, the overall effect of the $2 h-1 p$ configurations is AF in AF systems or $\mathrm{F}$ in ferromagnetic systems, where the kinetic exchange term is inexistent or very small.

A computational experiment has been carried out to

TABLE VII. Computational experiment to illustrate the modulation of the $2 h-1 p$ excitations ranging from $\mathrm{AF}$ to $\mathrm{F}$ contributions to the magnetic coupling constant. Effect of the out-of-plane $\mathrm{H}$ displacement on $J\left(\mathrm{~cm}^{-1}\right)$ for system 4 . The right column corresponds to the real structure.

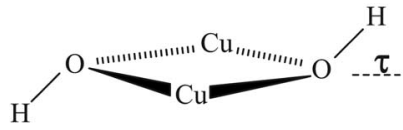

\begin{tabular}{|c|c|c|c|}
\hline$\tau^{(0)}$ & 0 & 38 & $61.5^{\mathrm{a}}$ \\
\hline CAS & 33 & 33 & 33 \\
\hline $\mathrm{CAS}+\mathrm{S}$ & 43 & 57 & 73 \\
\hline DDCI2 & 37 & 49 & 63 \\
\hline$+2 h-1 p$ & -39 & 79 & 195 \\
\hline$+1 h-2 p$ & 36 & 47 & 57 \\
\hline DDCI & 54 & 91 & 157 \\
\hline
\end{tabular}

${ }^{\mathrm{a}}$ Reference 43 . 
check the modulation of the $2 h-1 p$ effect (AF to F) depending on the nature of the system. As previously reported, the out-of-plane displacement $(\tau)$ of the hydrogen atoms in system 4 seems to favor the ferromagnetic character of the interaction. ${ }^{71-73}$ Two model structures with $\tau=0$ and $38^{\circ}$ have been calculated giving decreasing $\mathrm{F}$ coupling with decreasing out-of-plane distortion. The CI results given in Table VII illustrate how the $2 h-1 p$ acting on the DDCI2 space can introduce a F or AF contribution depending on the nature of the system (AF contribution for $\tau=0$; F contribution for $\tau \neq 0$ ).

\section{Damping effect of the $1 \boldsymbol{h}-2 p$ excitations}

In contrast to $2 h-1 p$ excitations, the $1 h-2 p$ determinants have only a secondary impact on the $J$ value, as can be observed from the partial results reported in Table V.

(i) When acting on the bare valence CAS, a rather small $\mathrm{F}$ effect is observed (see column $\Delta J$ on Table V). This effect can be rationalized as a decrease in the effective hopping integral $t_{a b}$ since the coupling between the $N$ and $I$ configurations mediated by the $1 h-2 p$ excitations: $N \leftrightarrow 1 h-2 p \leftrightarrow I$ has an opposite sign to the interaction through the $2 h-1 p$ excitations: $N \leftrightarrow 2 h-1 p \leftrightarrow I$.

(ii) When acting on the CAS $+\mathrm{S}$ or DDCI2 spaces the $1 h-2 p$ excitations are responsible for small $\mathrm{F}$ corrections on AF systems, quite close at both levels. Notice that for F systems, the direct effect of the $1 h-2 p$ excitations acting on $\mathrm{CAS}+\mathrm{S}$ or DDCI2 spaces is practically null, although with opposite sign. Unlike the $2 h-1 p$ excitations, the $1 h-2 p$ configurations do not introduce any significant effect by themselves, only small modifications of $J$ are obtained when adding them to $\mathrm{CAS}+\mathrm{S}$ or DDCI2 levels.

(iii) When acting together with $2 h-1 p$ excitations, they produce a damping of the global $2 h-1 p$ effect, working on the opposite direction that this last class does. That is, the $1 h-2 p$ class introduces a $\mathrm{F}$ contribution on $\mathrm{AF}$ systems, but AF one on $\mathrm{F}$ compounds. This effect is evidenced by comparing DDCI with DDCI2 $+2 h 1 p$ (or $\mathrm{CAS}+\mathrm{S}+2 h-1 p+1 h-2 p$ versus $\mathrm{CAS}+\mathrm{S}$ $+2 h-1 p)$ results in Table V.

(iv) Regarding the singlet and triplet wave functions, adding $1 h-2 p$ configurations produces a non-negligible reduction in the $C_{I} / C_{N}$ ratio in the singlet state as well as a significant drop in the weight of the LMCT forms (see Table VI), which correlates with the F correction of the $J$ value.

The interference between $1 h-2 p$ and $2 h-1 p$ excitations cannot be explained at low perturbation orders, as in the simple mechanism $N \leftrightarrow 1 h-2 p \leftrightarrow I \leftrightarrow 1 h-1 p \leftrightarrow N$, since as discussed above only very small effects are observed when simply adding both types of excitations to the CAS. It can only be attributed to complex high-order mechanisms as those discussed in Sec. VI A to interpret the role of $2 h-1 p$ excitations coupled to similar mechanisms involving the $1 h-2 p$ class.

A careful examination of the amplitude contours of the natural magnetic MOs calculated at different CI levels confirms the already observed increase in the localization on the ligands when correlation effects are included. ${ }^{70}$ This effect follows the sequence DDCI $2+2 h-1 p>$ DDCI $>$ DDCI2 $+1 h-2 p$, which is in line with the changes observed in the weight of the LMCT configurations on the correlated wave function and suggests that the $1 h-2 p$ excitations decrease delocalization. For system $\mathbf{1}, \mathrm{La}_{2} \mathrm{CuO}_{4}$, the largest coefficient out of the CAS concerns a LMCT configuration in the singlet state from the ungerade MO centered on the $2 p$ atomic orbital of the bridging ligand to the ungerade magnetic MO. Its coefficient is 0.0300 at the DDCI2 level, 0.1615 when adding only the $2 h-1 p$ excitations, 0.0227 when adding only the $1 h-2 p$, and 0.1250 at the DDCI level. This conflictive impact of $2 h-1 p$ and $1 h-2 p$ excitations on the LMCT component of the wave function is consistent with their opposite effect on the magnetic coupling amplitude. This is consistent with the qualitative consideration that the tails of the magnetic orbitals on the bridging ligands are implied in direct exchange, spin polarization, and kinetic exchange mechanisms.

\section{FINAL DISCUSSION}

The numerically crucial contribution of the two hole-one particle $(2 h-1 p)$ excitations in the calculation of the magnetic coupling constant between two $\mathrm{Cu}\left(d^{9}\right)$ magnetic centers has been reexamined. Their role is illustrated on a series of nine systems, which are either molecular complexes or fragments of magnetic lattices, either ferro- or antiferromagnetically coupled. The present results show that the previous interpretation $^{33}$ based on second-order perturbative arguments is not relevant since the effect of this class of excitations on the coupling constant is of higher-order character, which explains the rather poor performances of CAS-based second-order treatments when the CAS is limited to the two magnetic electrons in the two magnetic orbitals. As shown in the present work, in AF systems these $2 h-1 p$ configurations lead to an increase in the weight of one hole, $1-h$, i.e., LMCT configurations. This increase can be interpreted as a correlation-induced delocalization of the magnetic orbitals on the ligands. This causes the magnetic orbitals to take larger tails on the bridging ligands and therefore both direct and kinetic exchanges are larger. This results in an increase in the absolute value of $J$ when compared to DDCI2 values, whatever its sign. This correlation-induced delocalization becomes evident looking at the natural magnetic orbitals obtained at the DDCI level, which have been extensively analyzed in Ref. 70. These orbitals, which incorporate the effect of the dynamical correlation, are more diffuse than those obtained at the CAS mean-field level. This phenomenon is not limited to $\frac{1}{2}$ spin systems, but it concerns as well higher spin metallic ions. However, since the delocalization is caused by mechanisms that occur at high perturbation orders, these effects cannot be incorporated from a second-order expansion from a mean-field description of the minimal multireference space. This fact is not a particular effect of magnetic systems but a more general fact also observed in other fields. ${ }^{74}$

The present analysis indicates that variational wave 
function methods are the best choice for the calculation of the magnetic coupling constants when available. However, the procedure becomes very expensive from a computational point of view when several magnetic centers are involved, and in particular for particles with spin $S>\frac{1}{2}$. To overcome this constraint it has been suggested in the past that the dominant effect of $2 h-1 p$ and eventually $1 h-2 p$ excitations might be captured by enlarging the CAS with inactive occupied (and virtual) orbitals (CASext) and performing the corresponding CASext+S, CASext-DDCI2, or CASext+PT2 calculations, which are of lower computational cost than the minimal CAS DDCI one. This procedure faces first the problem of the definition of the holes which would play the dominant role in the $2 h-1 p$ class. Several attempts have been reported. The bridging ligand MOs may be selected from canonical MOs on the basis of their participation in the energy difference (dedicated MOs), ${ }^{75,76}$ of their spatial localization, ${ }^{77}$ or by projecting the tails of the CASSCF magnetic MOs in the space of the doubly occupied MOs, as done by Bordas et al..$^{51}$ Only a part of the $2 h-1 p$ effects are included by this procedure but the results show a systematic overestimation of the magnetic coupling, which is in line with the important damping role of the missing $1 h-2 p$ excitations. A detailed and critical analysis of the results coming from this strategy will be reported in a further work.

However, even assuming a certain overestimation of $J$ amplitudes, it seems clear that the use of extended CAS, both in perturbative or variational schemes, would be rapidly blocked by the size of the active space [for instance, a tetranuclear cluster of $\mathrm{Ni}$ (II) requires at least $16 d$ orbitals and a set of occupied orbitals centered on the bridging ligands]. In this frame, current methodological efforts concern the development of new approaches dealing with minimal CAS DDCI method at low cost. Works are in progress to reduce the cost of minimal CAS DDCI procedure based on the use of localized orbitals and topologically restricted CI calculations with promising results on the systems considered. ${ }^{78}$

Alternatively, solutions combining variational and perturbative strategies could also be useful. Starting from a minimal CAS involving the magnetic electrons and orbitals, the need to treat variationally the most numerous excitations of the DDCI space, namely, $2 h-1 p$ and $1 h-2 p$ classes, prevents a procedure consisting of adding the second-order perturbative effect of these two classes to the DDCI2 energies. However, a procedure based on a perturbative selection of the most important configurations of these classes and their addition to the DDCI2 space could offer a practical computational solution. This strategy has been employed in the past for the evaluation of $J$ on $\mathrm{La}_{2} \mathrm{CuO}_{4}$, providing values in good agreement with DDCI ones at a quite reduced computational cost, ${ }^{23}$ and deserves a deeper exploration in the future. A similar proposal is offered in the ORCA package. ${ }^{17,79}$

\section{ACKNOWLEDGMENTS}

The authors want to acknowledge the financial support from different institutions. R.C. and C.J.C. are indebted to Ministerio de Ciencia e Innovación of Spain (Project Nos. CTQ2008-06644-C02-01 and CTQ2008-06644-C02-02).
R.C. and D.T. also acknowledge AGAUR of the Generalitat de Catalunya (Grant No. 2005SGR-00104). This work has received the support of the European Union through the Cost action D37/0004/0006 and of the French-Italian cooperation through the PICS project No. 4263. The Laboratoire de Chimie et Physique Quantiques is "Unite Mixte de Recherche" UMR 5626 of the CNRS. This paper is dedicated to Alessandro Bencini for his crucial contributions to molecular magnetism and its interpretation. As theoreticians, we want to stress his role in an open and fair dialog between DFT and wave function based approaches.

${ }^{1}$ P. W. Anderson, Phys. Rev. 79, 350 (1950).

${ }^{2} \mathrm{P}$. W. Anderson, in Theory of the Magnetic Interaction: Exchange in Insulators and Superconductors, Solid State Physics Vol. 14, edited by F. Turnbull, and F. Seitz, (Academic, New York, 1963), p. 99.

${ }^{3}$ W. Van den Heuvel and L. F. Chibotaru, Phys. Rev. B 76, 104424 (2007).

${ }^{4}$ F. Illas, I. D. R. Moreira, C. de Graaf, and V. Barone, Theor. Chem. Acc. 104, 265 (2000).

${ }^{5}$ C. Adamo, V. Barone, A. Bencini, R. Broer, M. Filatov, N. M. Harrison, F. Illas, J.-P. Malrieu, and I. D. R. Moreira, J. Chem. Phys. 124, 107101 (2006).

${ }^{6}$ E. Ruiz, J. Cano, S. Alvarez, and V. Polo, J. Chem. Phys. 124, 107102 (2006).

${ }^{7}$ A. Bencini, Inorg. Chim. Acta 361, 3820 (2008).

${ }^{8} \mathrm{Ph}$. de Loth, P. Cassoux, J.-P. Daudey, and J.-P. Malrieu, J. Am. Chem. Soc. 103, 4007 (1981).

${ }^{9}$ M. F. Charlot, M. Verdaguer, Y. Journaux, Ph. de Loth, and J.-P. Daudey, Inorg. Chem. 23, 3802 (1984).

${ }^{10} \mathrm{Ph}$. de Loth, J.-P. Daudey, H. Astheimer, L. Walz, and W. Haase, J. Chem. Phys. 82, 5048 (1985).

${ }^{11}$ J.-P. Daudey, Ph. de Loth, and J.-P. Malrieu, in Magneto-Structural Correlation in Exchange Coupled Systems, NATO Advanced Studies Series Vol. 140, edited by R. D. Willett, D. Gatteschi, and O. Kahn (Reidel, Dordrecht, 1985), p. 87.

${ }^{12} \mathrm{Ph}$. de Loth, P. Karafiloglou, J.-P. Daudey, and O. Kahn, J. Am. Chem. Soc. 110, 5676 (1988).

${ }^{13}$ J.-P. Malrieu, J. Chem. Phys. 47, 4555 (1967).

${ }^{14}$ R. Broer and W. J. A. Maaskant, Chem. Phys. 102, 103 (1986).

${ }^{15}$ J. Miralles, J.-P. Daudey, and R. Caballol, Chem. Phys. Lett. 198, 555 (1992).

${ }^{16}$ J. Miralles, O. Castell, R. Caballol, and J.-P. Malrieu, Chem. Phys. 172, 33 (1993).

${ }^{17}$ F. Neese, J. Chem. Phys. 119, 9428 (2003).

${ }^{18}$ F. Neese, T. Petrenko, D. Ganyushin, and G. Olbrich, Coord. Chem. Rev. 251, 288 (2007).

${ }^{19}$ V. Barone, I. Cacelli, A. Ferretti, and M. Girlanda, J. Chem. Phys. 128, 174303 (2008).

${ }^{20}$ O. Castell, R. Caballol, V. M. García, and K. Handrick, Inorg. Chem. 35, 1609 (1996).

${ }^{21}$ J. Cabrero, N. Ben Amor, C. de Graaf, F. Illas, and R. Caballol, J. Phys. Chem. A 104, 9983 (2000).

${ }^{22}$ N. Suaud and M. B. Lepetit, Phys. Rev. B 62, 402 (2000).

${ }^{23}$ C. J. Calzado, J. F. Sanz, and J.-P. Malrieu, J. Chem. Phys. 112, 5158 (2000).

${ }^{24}$ C. de Graaf, C. Sousa, I. de P. R. Moreira, and F. Illas, J. Phys. Chem. A 105, 11371 (2001).

${ }^{25}$ J. Cabrero, C. de Graaf, E. Bordas, R. Caballol, and J.-P. Malrieu, Chem.Eur. J. 9, 2307 (2003).

${ }^{26}$ C. J. Calzado, C. de Graaf, E. Bordas, R. Caballol, and J.-P. Malrieu, Phys. Rev. B 67, 132409 (2003).

${ }^{27}$ C. J. Calzado, S. Evangelisti, and D. Maynau, J. Phys. Chem. A 107, 7581 (2003).

${ }^{28}$ N. Queralt, D. Taratiel, C. de Graaf, R. Caballol, R. Cimiraglia, and C. Angeli, J. Comput. Chem. 29, 994 (2008).

${ }^{29}$ C. de Graaf, L. Hozoi, and R. Broer, J. Chem. Phys. 120, 961 (2004).

${ }^{30}$ C. Aronica, E. Jeanneau, H. El Moll, D. Luneau, B. Gillon, A. Goujon, A. Cousson, M. A. Carvajal, and V. Robert, Chem.-Eur. J. 13, 3666 (2007).

${ }^{31}$ J. B. Rota, L. Norel, C. Train, N. Ben Amor, D. Maynau, and V. Robert, J. Am. Chem. Soc. 130, 10380 (2008). 
${ }^{32}$ N. Guihery, V. Robert, and F. Neese, J. Phys. Chem. A 112, 12975 (2008).

${ }^{33}$ C. J. Calzado, J. Cabrero, J. P. Malrieu, and R. Caballol, J. Chem. Phys. 116, 2728 (2002).

${ }^{34}$ C. J. Calzado, J. Cabrero, J. P. Malrieu, and R. Caballol, J. Chem. Phys. 116, 3985 (2002).

${ }^{35}$ P. E. Sulewski, P. A. Fleury, K. B. Lyons, S. W. Cheong, and Z. Fisk, Phys. Rev. B 41, 225 (1990).

${ }^{36}$ R. P. Singh, P. A. Fleury, K. B. Lyons, and P. C. Sulewski, Phys. Rev. Lett. 62, 2736 (1989).

${ }^{37}$ G. Aeppli, S. M. Hayden, H. A. Mook, Z. Fisk, S. W. Cheong, D. Rytz, J. P. Remeika, G. P. Espinosa, and A. S. Cooper, Phys. Rev. Lett. 62 2052 (1989)

${ }^{38}$ S. M. Hayden, G. Aeppli, R. Osborn, A. D. Taylon, T. G. Perring, S. W. Cheong, and Z. Fisk, Phys. Rev. Lett. 67, 3622 (1991).

${ }^{39}$ M. Greven, R. J. Birgeneau, Y. Endoh, M. A. Kastner, M. Matsuda, and G. Shirane, Z. Phys. B 96, 465 (1995).

${ }^{40}$ T. P. Mitchell, W. H. Bernard, and J. R. Wasson, Acta Crystallogr., Sect. B: Struct. Crystallogr. Cryst. Chem. 26, 2096 (1970).

${ }^{41}$ B. J. Cole and L. V. H. Brumage, J. Chem. Phys. 53, 4718 (1970).

${ }^{42}$ R. J. Majeste and E. A. Meyers, J. Phys. Chem. 74, 3497 (1970).

${ }^{43}$ V. H. Crawford, H. W. Richardson, J. R. Wasson, D. J. Hodgson, and W. E. Hatfield, Inorg. Chem. 15, 2107 (1976).

${ }^{44}$ R. D. Willett, in Magneto-Structural Correlation in Exchange Coupled Systems, NATO Advanced Studies Series. C Vol. 140, edited by R. D. Willett, D. Gatteschi, and O. Kahn (Reidel, Dordrecht, 1985), p. 389.

${ }^{45}$ C. Chow and R. D. Willett, J. Chem. Phys. 59, 5903 (1973).

${ }^{46}$ B. Scott and R. D. Willett, Acta Crystallogr., Sect. C: Cryst. Struct. Commun. 47, 435 (1991).

${ }^{47}$ G. Maass, B. Gerstein, and R. D. Willett, J. Chem. Phys. 46, 401 (1967).

${ }^{48}$ G. O'Bannon and R. D. Willett, Inorg. Chim. Acta 53, 6131 (1983).

${ }^{49}$ P. Chaudhuri, K. Oder, K. Wieghardt, B. Nuber, and J. Weiss, Inorg. Chem. 25, 2818 (1986).

${ }^{50}$ T. R. Felthouse, E. J. Laskowski, and D. N. Hendrickson, Inorg. Chem. 16, 1077 (1977).

${ }^{51}$ E. Bordas, R. Caballol, C. de Graaf, and J.-P. Malrieu, Chem. Phys. 309, 259 (2005).

${ }^{52}$ Z. Barandiarán and L. Seijo, Can. J. Chem. 70, 409 (1992).

${ }^{53}$ P.-O. Widmark, P.-A. Malmqvist, and B. O. Roos, Theor. Chim. Acta 77 , 291 (1990).

${ }^{54}$ P.-O. Widmark, B. J. Persson, and B. O. Roos, Theor. Chim. Acta 79, 419 (1991).

${ }^{55}$ K. Pierloot, B. Dumez, P.-O. Widmark, and B. O. Roos, Theor. Chim.
Acta 90, 87 (1995)

${ }^{56}$ CASDI program: package developed at the Laboratoire de Physique Quantique, Université Paul Sabatier, Toulouse, France; N. Ben Amor and D. Maynau, Chem. Phys. Lett. 286, 211 (1998).

${ }^{57}$ G. Karlström, R. Lindh, P.-Å. Malmqvist, B. O. Roos, U. Ryde, V. Veryazov, P.-O. Widmark, M. Cossi, B. Schimmelpfennig, P. Neogrady, and L. Seijo, Comput. Mater. Sci. 28, 222 (2003).

${ }^{58}$ C. Angeli, R. Cimiraglia, S. Evangelisti, T. Leininger, and J.-P. Malrieu, J. Chem. Phys. 114, 10252 (2001).

${ }^{59}$ C. Angeli, R. Cimiraglia, and J.-P. Malrieu, Chem. Phys. Lett. 350, 297 (2001).

${ }^{60}$ C. Angeli, R. Cimiraglia, and J.-P. Malrieu, J. Chem. Phys. 117, 9138 (2002).

${ }^{61}$ The NEVPT2 calculations have been performed with a computational package developed at the University of Ferrara.

${ }^{62}$ L. Brillouin, J. Phys. (Paris) 3, 373 (1932).

${ }^{63}$ B. Levy and G. Berthier, Int. J. Quantum Chem. 2, 307 (1968).

${ }^{64}$ C. J. Calzado, J.-P. Malrieu, and J. F. Sanz, J. Phys. Chem. A 102, 3659 (1998).

${ }^{65}$ J.-P. Malrieu, N. Guihéry, C. J. Calzado, and C. Angeli, J. Comput. Chem. 28, 35 (2007).

${ }^{66}$ C. Angeli, R. Cimiraglia, and J.-P. Malrieu, J. Chem. Educ. 85, 150 (2008).

${ }^{67}$ C. Angeli, A. Cavallini, and R. Cimiraglia, J. Chem. Phys. 128, 244317 (2008).

${ }^{68}$ C. Angeli, C. J. Calzado, R. Cimiraglia, and J.-P. Malrieu, Chem. Phys. 124, 234109 (2006).

${ }^{69}$ C. Angeli, B. Bories, A. Cavallini, and R. Cimiraglia, J. Chem. Phys. 124, 054108 (2006).

${ }^{70}$ J. Cabrero, C. J. Calzado, D. Maynau, R. Caballol, and J. P. Malrieu, J. Phys. Chem. A 106, 8146 (2002).

${ }^{71}$ E. Ruiz, P. Alemany, S. Alvarez, and J. Cano, J. Am. Chem. Soc. 119, 1297 (1997).

${ }^{72}$ Q. Ren, Z. Chen, and L. Zhang, Chem. Phys. Lett. 364, 475 (2002).

${ }^{73}$ L. Gutierrez, G. Alzuet, J. A. Real, J. Cano, J. Borras, and A. Castineiras, Eur. J. Inorg. Chem. 2094 (2002).

${ }^{74}$ C. Angeli, J. Comput. Chem. 30, 1319 (2009)

${ }^{75}$ C. J. Calzado and J.-P. Malrieu, Eur. Phys. J. B 21, 375 (2001).

${ }^{76}$ C. J. Calzado and J.-P. Malrieu, Phys. Rev. B 63, 214520 (2001).

${ }^{77}$ A. Gellé, M. L. Munzarová, M. B. Lepetit, and F. Illas, Phys. Rev. B 68, 125103 (2003).

${ }^{78}$ D. Maynau, S. Hoyau, and J. P. Malrieu (in preparation).

${ }^{79}$ F. Neese, ORCA 2.6.35, http://www.thch.uni-bonn.de/tc/orca, 2009 